the shape and volume of gastrointestinal organs and localization of the lesion. The accurate localization of a malignant lesion within the global view of the stomach is crucial for gastric surgeons to make a clinical decision of the operative procedure. Further, the shape of gastrointestinal organs possibly associates with some abdominal symptoms or disorders. Structure from Motion ( $\mathrm{SfM}$ ) is a method to recover $3 \mathrm{D}$ scene structure and camera motion from multiple images. SfM may be applied to endoscopy in order to reconstruct the shape of gastrointestinal organs. We aimed to reconstruct the $3 \mathrm{D}$ model of the whole stomach from standard endoscopic video image using SfM.

Methods Seven participants underwent gastroscopy under sedation for screening upper gastrointestinal diseases. The endoscope video was captured using a standard endoscope system. The video data was saved as an AVI format in 30 frames per second with full HD resolution. All endoscopic video was converted to RGB frames. The red channel of the extracted RGB frames were put to the SfM, where feature extraction, matching, pose estimation, and feature points triangulation were performed to generate a sparse $3 \mathrm{D}$ point cloud. Poisson surface reconstructed was performed to construct a 3D mesh model of the stomach. Finally, the texture of the endoscopic images was applied to the generated 3D mesh model to add more visual detail.

Results Most 3D point clouds were extracted from the red channel of the endoscopic images with the indigo carmine dye (figure 1). 3D meshes and texture representation resembling the whole shape of a stomach were generated from the cloud model (figure 2). Gastric ulcer lesion was clearly localized and reconstructed in one subject.

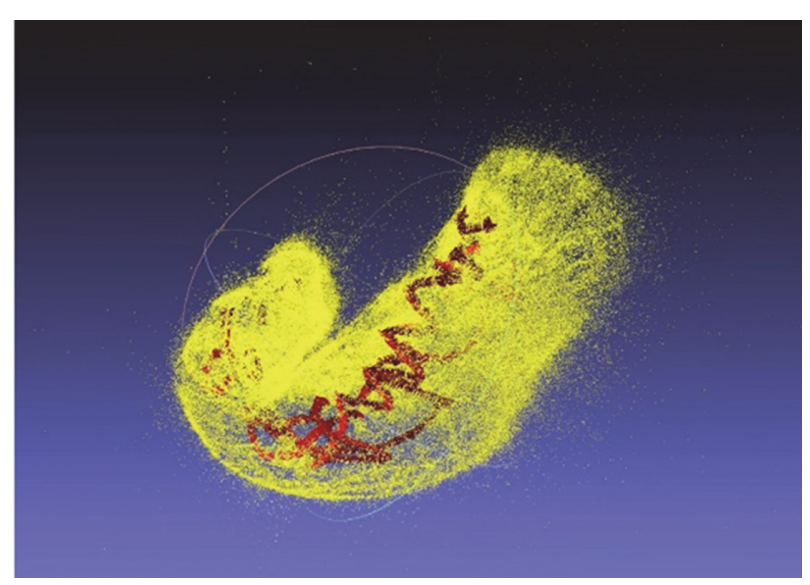

Abstract IDDF2020-ABS-0068 Figure 1
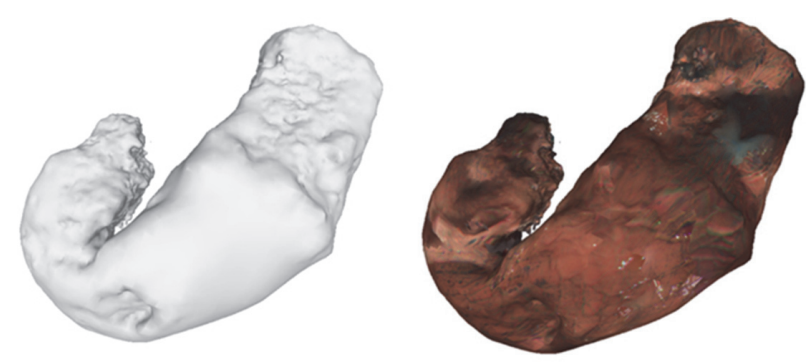

Abstract IDDF2020-ABS-0068 Figure 2
Conclusions Our study found that $3 \mathrm{D}$ reconstruction of the whole stomach can be achieved from a standard endoscopic video image using SfM. Furthermore, gastric ulcer lesion was also localized and reconstructed in $3 \mathrm{D}$ reconstruction model. Our future work will be focused on the clinical significance of our proposed method. We will try to evaluate the clinical usefulness of this method for the patients undergone surgery for early gastric cancers.

\section{IDDF2020-ABS-0070 PROTEOMIC ANALYSIS OF COLORECTAL CANCER WITH BRAFV600E MUTATION}

Biting Zhou*, Kailun Xu, Yingkuan Shao, Xi Zheng, Shu Zheng. Cancer Institute (Key Laboratory of Cancer Prevention and Intervention, China National Ministry of Education, Key Laboratory of Molecular Biology in Medical Sciences, China), The Second Affiliated Hospital, Zhejiang University School of Medicine, China

\subsection{6/gutjnl-2020-IDDF.59}

Background Proteomics provides new insights on tumorigenesis and progression of colorectal cancer (CRC) dependent of genomics and transcriptomics. BRAFV600E mutation is an unfavorable prognosis biomarker, occurring in about $5-20 \%$ CRC. In this study, we aimed to figure out the molecular characteristics in CRC with BRAFV600E mutation in proteomics.

Methods Based on a novel approach combining pressure cycling technology with the data-independent acquisition of mass spectrometry, thousands of peptides were obtained from formalin-fixed paraffin-embedded tumor tissue samples from 249 patients with colon cancer during 2009-2012 and then were processed through OpenSWATH to get the protein matrix. Differentially expressed proteins were analyzed by t-test ( $\mathrm{p}$-value $<0.05$, fold-change $>1.5 /<2 / 3$ ). BRAFV600E mutation status was tested by amplificationrefractory-mutation-system-based PCR assays. Biological function and pathway enrichment analysis were performed by STRING.

Results 27 patients were proved BRAFV600E mutation, indicating $10.8 \%$ occurrence rate in our CRC cohort. 276 differentially expressed proteins were obtained, including 243

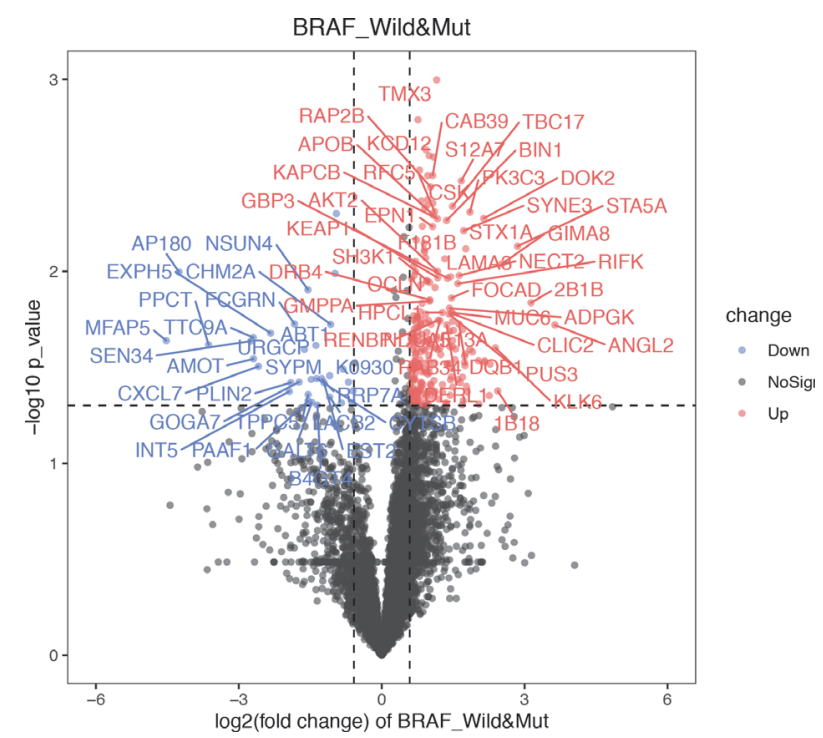

Abstract IDDF2020-ABS-0070 Figure 1 


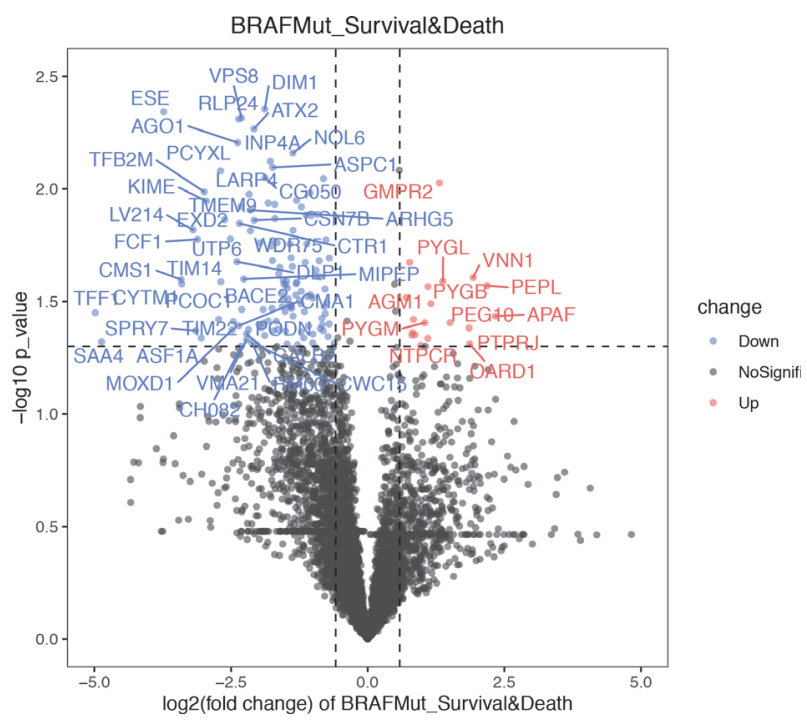

Abstract IDDF2020-ABS-0070 Figure 2

upregulated and 33 downregulated in BRAFV600E mutation versus matched wild type (figure 1). The most significant biological processes for overexpressed proteins are interferon-gamma-mediated signaling pathway and antigen processing and presentation of exogenous peptide antigen, while for downregulated proteins is ncRNA metabolic process. In BRAFV600E mutated samples, 151 differentially expressed proteins were obtained, including 131 downregulated and 20 upregulated from patients' overall survival less than 5 years versus exceeding 5 years (figure 2). The overrepresented biological process and molecular functions are leukocyte mediated immunity and SHG alpha-glucan phosphorylase activity for overexpressed proteins, while ribosome biogenesis and RNA binding for downregulated proteins.

Conclusions Based on the general and primary proteomic analysis, we found that BRAFV600E mutated CRC exhibited immune-related characteristics, and the poor prognosis might relate to the abnormal metabolic process. Relevant mechanisms and pathways will be studied in further research.

\section{IDDF2020-ABS-0072 EFFECT OF COMPREHENSIVE NURSING INTERVENTION ON PERIOPERATIVE PSYCHOLOGICAL AND STRESS STATUS OF ERCP}

Wen-hui Tan*, Xiao-qin Zhang, Dong-hong He, Fang Wang. Guangdong Second Provincial General Hospital, China

\subsection{6/gutjnl-2020-IDDF.60}

Background To investigate the effect of comprehensive nursing intervention on the psychological and stress status of ERCP patients during the perioperative period.

Methods A total of 100 patients who underwent ERCP in our Endoscopy Center from July 2018 to July 2019 were enrolled for the research. The first 50 patients were selected as a control group and given routine care in chronological order, while the last 50 patients were selected as observation group who were implemented perioperative comprehensive nursing intervention on a routine basis. A psychological evaluation was performed on both groups of patients after operation.

Results The anxiety score $(14.01 \pm 0.11)$ and depression score $(15.23 \pm 1.48)$ of the observation group were significantly lower than those of the control group $(\mathrm{P}<0.05)$; stress status of the observation group (slightly 30 cases (60.00\%), moderate 20 cases(40.00\%)) was significantly different from those of the control group $(\mathrm{P}<0.05)$; the complication rate in the observation group was $4.00 \%$ (1 case of acute pancreatitis, 1 case of cholangitis) and it was significantly lower than that in the control group $(\mathrm{P}<0.05)$.

Conclusions The implementation of comprehensive nursing intervention for ERCP patients during perioperative period can effectively improve the negative emotions of patients, improve the stress status of patients, reduce the incidence of complications, and be beneficial to postoperative recovery.

\section{IDDF2020-ABS-0074 ANALYSIS OF COAGULATION FUNCTION IN PATIENTS WITH INFLAMMATORY BOWEL DISEASE}

Yi Yu*. The Second Affiliated Hospital of Guangzhou University of Chinese Medicine, China

\subsection{6/gutjint-2020-IDDF.61}

Background To study the related factors of hypercoagulability in patients with inflammatory bowel disease (IBD) and their relationship with CRP, ESR and D-D, and to screen out the population with the highest risk of hypercoagulation, so as to prevent the formation of thrombosis in the early stage.

Methods We directly extract data from the MIMIC-III and Philips eICU collaboration databases. 739 cases were met; the diagnostic standard was included and divided into UC group and $\mathrm{CD}$ group. The patients were divided into groups of 0 20 years old, 20-40 years old, 40-60 years old and 60-80 years old, and divided into male and female according to gender. The patients with UC were divided into mild group, moderate group and severe group according to the improved Truelove and Witts severity. The patients hospitalized with polyps of digestive diseases were chosen as normal control group. Record data about blood routine examination, coagulation function and D-D were also collected.

Results There were statistical differences in TT and FIB between patients with IBD and those with polyps $(\mathrm{P}<0.05)$, but no statistical differences in APTT. There was no statistical difference between patients with UC and patients with $\mathrm{CD}$ in TT, FIB and APTT. There was no statistical difference in the analysis of TT and FIB among IBD patients in terms of age. There were no statistical differences in TT and FIB among UC patients in terms of gender and age; FIB was statistically different in the disease severity with the active phase, while TT was not statistically different.

\section{Conclusions}

1. IBD patients have higher coagulation status than normal people. The hypercoagulability of IBD patients was correlated with ESR, but not with CRP and D-D.

2. The higher the age of $\mathrm{CD}$ patients, the higher the risk of coagulation. The risk of hypercoagulability in UC patients was independent of gender and age; The more serious the condition was in the active stage, the more significant the hypercoagulability was.

3. The hypercoagulability of UC patients has little correlation with ESR and D-D, but no correlation with CRP. 